\title{
TRANSVERSE COMBINING OF FOUR BEAMS IN MBE-4*
}

\author{
C.M. Celata, W. Chupp, A. Faltens, W.M. Fawley, W. Ghiorso, K.D. Hahn, E. Henestroza, C. Peters, and \\ P. Seidl, Lawrence Berkeley Laboratory, University of California, Berkeley, CA 94720
}

\begin{abstract}
Transverse beam combining is a cost-saving option employed in many designs for induction linac heavy ion fusion drivers. But resultant transverse emittance increase, due predominantly to anharmonic space charge forces, must be kept minimal so as not to sacrifice focusability at the target. A prototype combining experiment has been built, using the MBE-4 experiment. Four sources produce four 4 $\mathrm{mA} \mathrm{Cs}{ }^{+}$beams at $200 \mathrm{keV}$. The ion sources are angled toward each other, so that the beams converge. Focusing upstream of the merge consists of 4 quadrupoles and a final combined-function element (quadrupole $\&$ dipole). All lattice elements are electrostatic. Due to the small distance between beams at the last element $(\sim 2 \mathrm{~mm})$, the electrodes here are a cage of small wires, each at different voltage. The beams emerge into the 30 period transport lattice of MBE-4 where emittance growth due to merging, as well as the subsequent evolution of the distribution function, can be diagnosed. The combiner design, simulation predictions, and preliminary results from the experiment are presented.*
\end{abstract}

\section{MOTIVATION}

Transverse beam combining is an important cost-saving feature of standard driver designs for heavy ion fusion. At the low-energy end of a driver, electrostatic quadrupoles are used to focus each beam of the multiple-beam array. Voltage breakdown and economics considerations dictate a small aperture for these quadrupoles, and thus a large number of beams. At higher energies it is more economical to accelerate fewer fatter beams through large-aperture magnetic quadrupoles. Thus at some energy $\sim 100 \mathrm{MeV}$, transverse beam combining occurs.

Since space charge dominates beam dynamics for these intense beams, the interactions between particles during merging serve as a source of emittance growth, along with the usual "phase space filling" seen, for instance, in beam stacking in storage rings. As shown in previous work,transverse emittance growth is minimized by packing the beams as tightly as possible. Near the merge point the small space between beams makes it difficult to insert focusing structures with good field quality. The experimental challenge is to position the beams to sufficient accuracy to allow tight packing, and to keep them focused as their centroids converge.

\section{DESCRIPTION OF THE EXPERIMENT}

At Lawrence Berkeley Laboratory an experiment to demonstrate 4-to-1 transverse beam combining is being installed on the old MBE-4 accelerator. A "combiner", consisting of a Cs ${ }^{+}$source, $200 \mathrm{keV}$ diode, and focusing transport channel for each of the four $4 \mathrm{~mA}$ beams, replaces the old MBE-4 diode and matching system. The beamlines converge with angle of $6_{i}$ relative to the combiner centerline. A diagram of one of the beamlines is shown in Fig. 1. Four electrostatic quadrupoles, followed by an electrostatic combined-function quadrupole + dipole, are used to focus each beam and straighten its trajectory so that the beams emerge from the combiner almost parallel to the centerline. The design configuration for the beam cross sections as they emerge from the combiner is shown in Fig. 2. As can be seen, the cross section is $x-y$ asymmetric to allow for good packing of the elliptical beams. After the combiner the merged beam is transported (without acceleration) and diagnosed in one transport channel of the remaining 30 lattice periods of MBE4.

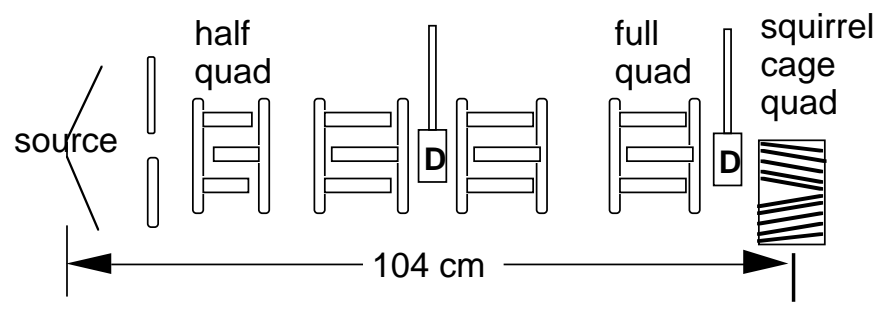

Figure 1. One combiner beamline. Diagnostics are on paddles marked "D".

Due to length restrictions, matching is done in the combiner, rather than in a separate matching system. For the same reason, the need for an initial dipole for each beam has been removed by aiming the sources toward a common point of convergence.

As the separation between beams decreases with distance down the combiner, the design of the lattice elements must change. The first three combiner quadrupoles are of standard design, and consist of circular electrodes, with the ratio of electrode radius to aperture set to minimize the lowest order 2D nonlinear field component-- the dodecapole. The first quadrupole is half the length of succeeding quads to facilitate matching. At the fourth quadrupole, the space between beams is too small for standard electrodes, so that small-aperture hyperbolic electrodes have been designed which both shield the beams from each other and produce minimal nonlinearity. Taking advantage of the elliptical shape of the beam, the electrodes in the focusing plane have also been moved closer to the beam to allow space for adjacent quadrupoles, thus making an asymmetric aperture. Voltages on the first four quadrupoles are all less than $5 \mathrm{kV}$.

\footnotetext{
* This work was supported by the Director, Office of Energy Research, Office of Fusion Energy, U.S. Dept. of Energy, under Contract No. DE-AC03-76SF00098.
} 


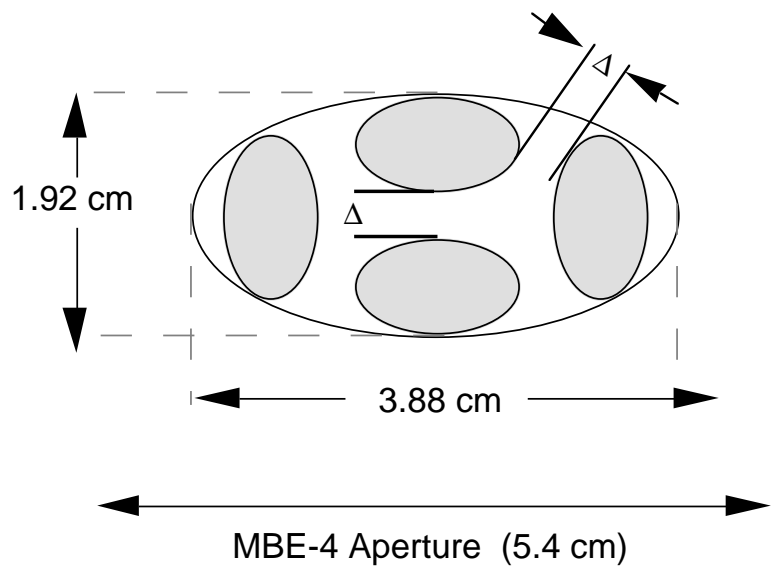

Figure 2. Design configuration of four beams as they emerge from combiner. Beam radii are 6.5 and $3.7 \mathrm{~mm} . \Delta=4 \mathrm{~mm}$.

The small spacing between the beams at the downstream end of the fifth lattice element does not allow adequate space for solid electrodes. Quadrupole and dipole fields are instead produced by surrounding the beams with an elliptical "squirrel cage" of $1 \mathrm{~mm}$ diameter wires, at a spacing of approximately $1 \mathrm{~mm}$, approximately parallel to the beam path. The voltage on each wire is set so that the wires approximately produce the correct Dirichlet boundary condition for the electric field, and therefore the correct field. Voltage differences of up to $1.5 \mathrm{kV}$ from wire to wire are needed. Figure 3 shows a transverse view of the squirrel cage element. Note that the cross section shrinks in a self-similar fashion as the beam centroids converge.

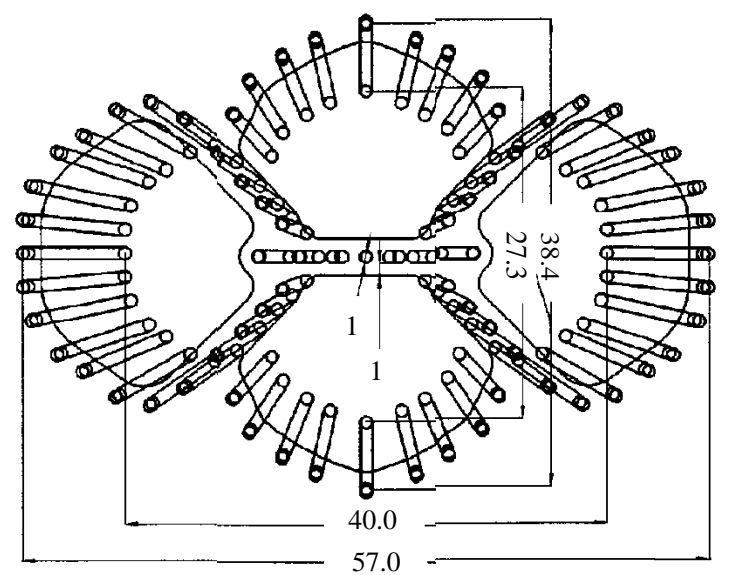

Figure 3. Transverse view of squirrel cage, showing wires converging as $\mathrm{z}$ increases. Dimensions are in millimeters.

Alignment is accomplished with sources mounted on gimbals which allow them to rotate about their centers, followed by articulation in $\mathrm{x}$ and $\mathrm{y}$ of the second quadrupole. Both operations can be done in vacuum. Since the beams emerge from the combiner separated by about $4 \mathrm{~mm}$, their clearance from the wires within the squirrel cage is only about a millimeter near the exit of the cage. Thus alignment must be correct to the sub-millimeter level.

\section{FIELDS OF THE SQUIRREL CAGE}

The 3D field of the squirrel cage has been calculated in order to evaluate the effects of fringe and anharmonic interior fields on the beams. In this initial calculation the wire leads were neglected, but no extension of the technique is needed to include them at a later time. Fields were calculated using the capacitive matrix technique. This is equivalent to modeling the wires (at constant voltage) as lines of charges. The charges were spaced at intervals, $\Delta \mathrm{z}$, of $1.25 \mathrm{~mm}$ along the 7 $\mathrm{cm}$ length of the cage. Elements of the capacitive matrix can be expressed as:

$$
C_{i j}=\frac{1}{\operatorname{Max}\left[\left|r_{i}-r_{j}\right|, r_{o}\right]},
$$

where $r_{i}$ and $r_{j}$ are the positions of two of the charges, and $r_{0}=\Delta z /(4 \log 2)$. The effective size of the charge, $r_{0}$, is chosen to produce as little variation in voltage along the wire as possible. Once the capacitive matrix is inverted, the charges can be determined, since $\mathrm{V}\left(\mathrm{r}_{\mathrm{i}}\right)=\Sigma \mathrm{C}_{\mathrm{ij}}{ }^{-1} \mathrm{Q}_{\mathrm{j}}$. The field is then determined using Coulomb's law.

Figure 4 shows the results of a multipole decomposition of the field about a parabolic approximation to the beam centroid orbit. The decomposition was done using the method of Hahn. The dominant nonlinear component through most of the cage is a sextupole. Near the ends of the cages the pseudo-octupole caused by the second derivative of the quadrupole moment becomes significant.

In order to obtain a measure of the emittance growth expected from the anharmonic fields, these fields were integrated along the approximate orbit of the beam centroid. When appropriately scaled, the gradient of this integrated value gives the net impulse in both $\mathrm{x}$ and $\mathrm{y}$ velocities as a function of location with respect to the beam centroid. The results show that over most of the aperture the calculated impulse in $d x / d z$ and $d y / d z$ is small when compared to the dipole deflection angle and comparable to the thermal angles of the incoming beam particles. Particles that come within $\sim 1.5 \mathrm{~mm}$ of the wires can receive larger impulses, but our calculations are less trustworthy in that regime due to the 1.25 $\mathrm{mm}$ spacing adopted for the charges used to simulate the charged wires. The changes in angle noted here are about an order of magnitude less than those expected during merging, indicating that the anharmonic squirrel cage fields are well within acceptable tolerance levels.

\section{SIMULATION RESULTS}

Transport through the entire combiner, and subsequent merging and transport of the merged beam have been simulated using the 2D particle-in-cell code HIBEAM. Four $4 \mathrm{~mA}$ beams of $\mathrm{Cs}^{+}$with initial normalized emittance of $2 \mathrm{x}$ $10^{-8} \mathrm{~m}$-rad, and energy of $200 \mathrm{keV}$ are assumed. A semigaussian initial distribution function is used for each beam-i.e., uniform spatial density and Maxwellian velocity distribution. One beam is followed where the beams are well separated (up to $8 \mathrm{~cm}$ before the squirrel cage), neglecting effects of other beams and quadrupoles. Image effects of the 
focusing structures included are calculated using the capacitive matrix technique. At the fourth quadrupole the surfaces of the electrodes are modeled as hyperbolic, as in the experiment, and the fields and image fields of adjacent quadrupoles are included, due to their proximity. Because of the 2D nature of the simulation, wires in the squirrel cage are modeled by dividing their length into 16 equal segments parallel to the centerline of the combiner, but with aperture equal to that of the real combiner wires at the center of the interval.

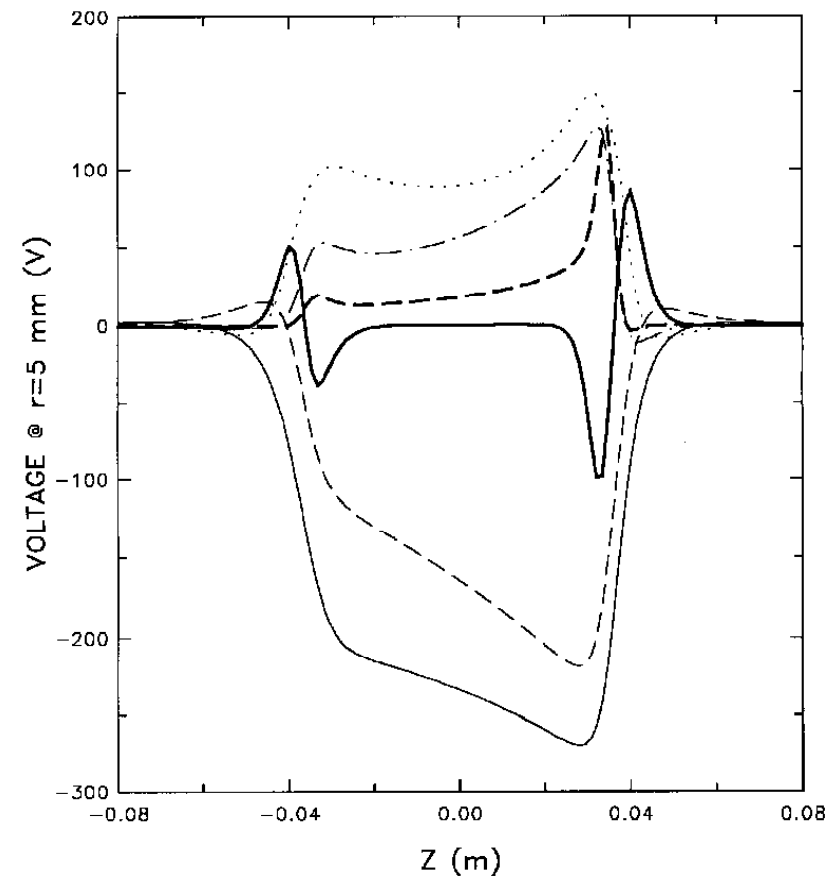

Figure 4. Squirrel cage multipole moments as a function of $\mathrm{z}$. At $\mathrm{z}=0$, in order of decreasing ordinate position, those shown are: $10 \mathrm{x}$ sextupole, $10 \mathrm{x}$ octupole, $10 \mathrm{x}$ pseudooctopole, $10 \times$ dodecapole, quadrupole, $0.1 \times$ dipole.

The resulting normalized emittance as a function of $\mathrm{z}$ is shown in Fig. 5. Image effects of the electrodes of the first three quadrupoles cause emittance growth of about $2 \times 10^{-9}$ $\mathrm{m}$-rad, while the field aberrations in the squirrel cage increase the emittance to approximately $3.5 \times 10^{-8} \mathrm{~m}$-rad. Asymmetry due to adjacent quadrupole electrodes at the fourth quadrupole results in negligible displacement of the centroid. During the merging process the emittance grows, to finally attain a value of $2.4 \times 10^{-7} \mathrm{~m}$-rad. Beam loss is negligible $(<0.02 \%)$. Without space charge, the expected final emittance would be approximately $1.1 \times 10^{-7} \mathrm{~m}$-rad, which demonstrates that this experiment is, like the driver, in the regime where both space charge and phase-space filling contribute to the final emittance growth. It should be noted that because of the relatively large spaces between the small beams in this experiment, emittance growth is proportionately bigger than it would be in a driver, where the much larger beams are expected to have approximately the same separation. Simulations have been done which show adequately low emittance growth for the case of the driver.

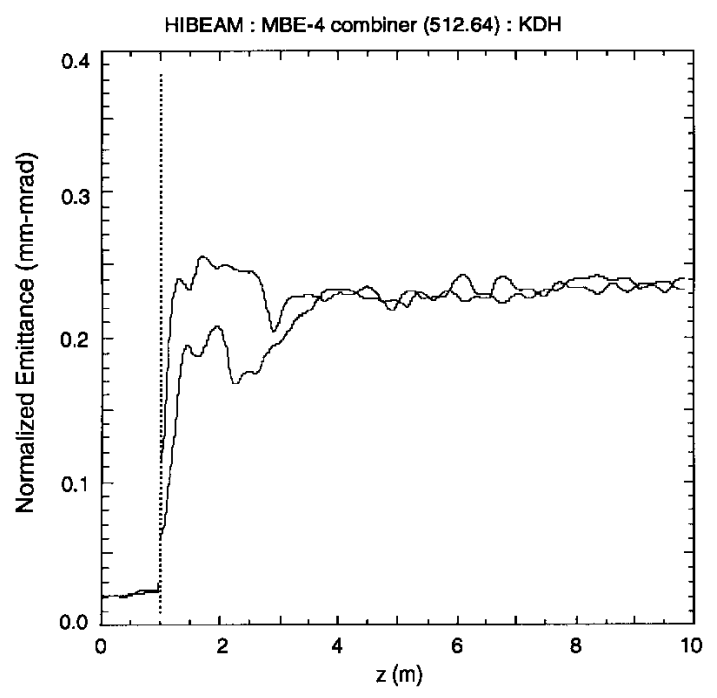

Figure 5. Normalized emittance as a function of $z$. Vertical line shows the end of the combiner.

\section{STATUS OF THE EXPERIMENT}

At present all components of the combiner have been fabricated and aligned with respect to the combiner center line, except the squirrel cage. The design is complete for this element as well. One of the sources, which are porous tungsten emitting surfaces saturated with an aqueous solution of $\mathrm{CsCO}_{3}$, has been installed in MBE-4. The effects of heat from the $1000^{\circ} \mathrm{C}$ emitting surface on alignment and on the source alignment adjustment motor have been found to be acceptable, but water cooling will be added to the driver motor support plates to improve the tolerance margin for the motors. A beam current of $6.7 \mathrm{~mA}$ at $198 \mathrm{keV}$ was measured $9.4 \mathrm{~cm}$ downstream of the source, in agreement with E-GUN calculations. This current will be adjusted using aperture plates just downstream of the diode during the actual experiment.

As time and resources allow, the diodes and first four quadrupoles for each beam will be installed in MBE-4, and experiments will be performed to measure the distribution function of the beams at the entrance to the squirrel cage. The squirrel cage will then be added, and the effect of the combiner on one beam measured, followed by full merging experiments.

\section{REFERENCES}

[1] C.M. Celata A. Faltens, D.L. Judd, L. Smith, and M.G. Tiefenback, Proc. of 1987 Part. Acc. Conf., Washington, D.C., 2 (1987), 1167

[2] Thomas J. Fessenden, Denis Keefe, Charles Kim, Hermann Meuth, and Anthony Warwick, Proc. of 1987 Part. Acc. Conf., Wahington, D.C., 2 (1987), 898.

[3] K. Hahn, Nucl. Instrum Meth. 338 (1994),161. 